\title{
The Electrocardiographic Holter Monitoring in Experimental Veterinary Practice
}

\author{
P. SCHEER ${ }^{1}$, P. SVOBODA ${ }^{2}$, M. SEPŠI ${ }^{3}$, K. JANEČKOVÁ ${ }^{1}$, J. DOUBEK $^{1}$ \\ ${ }^{1}$ Department of Physiology, Faculty of Veterinary Medicine, University of Veterinary and \\ Pharmaceutical Science Brno, Czech Republic, ${ }^{2}$ Trauma Hospital Brno and Department of \\ Traumatology, Faculty of Medicine, Masaryk University, Brno, Czech Republic, ${ }^{3}$ Faculty Hospital \\ Brno, Czech Republic
}

Received February 19, 2010

Accepted April 28, 2010

\section{Summary}

The long-term electrocardiographic recording with retrospective evaluation (Holter system) has been widely used not only in cardiology, but also in other disciplines of internal medicine and in pharmaceutical research. The Holter system can be used in mini-pig, sheep, dog, cat, rabbit, ferret, and rat. In this paper hardware, software, and anesthesia requirements are summarized with respect to the experimental work with various species. As the Holter systems work in bipolar mode, the use of bipolar leads in sagittal and transversal planes has been proved to be the most appropriate because of large amplitude of QRS complex and uncomplicated consequent automatic analysis of the record. In conclusion, Holter electrocardiography represents a simple and applicable method for monitoring the electrical activity of the heart in small animals' experimental studies.
\end{abstract}

\section{Key words}

ECG Holter recording $\bullet$ Rat $\bullet$ Ferret $\bullet$ Rabbit $\bullet$ Cat $\bullet$ Dog $\bullet$ Minipig • Sheep

\section{Corresponding author}

Peter Scheer, Department of Physiology, Faculty of Veterinary Medicine, University of Veterinary and Pharmaceutical Science Brno, Palackého st. 1-3, 61242 Brno, Czech Republic. E- mail: scheerp@vfu.cz

\section{Introduction}

Breakthrough idea of Norman Holter in the sixties of the twentieth century has been established as basic diagnostic method during last three decades. Technological development and design refined the method and made it user-friendly. Miniaturization of the recorder allowed the use of Holter method in veterinary and experimental practice not only in large, but also in small animals (Miller and Calvert 1992, Sleeper 2008, Reefmann et al. 2009).

The long-term electrocardiographic recording with retrospective evaluation has been widely used not only in cardiology, but also in other disciplines of internal medicine and in pharmaceutical research. The advantages of classical Holter electrocardiography (HECG) are automatic recording, reasonable price and practical availability, low operating costs and relatively good information acquisition. The method is limited by hardware of the recorder, analytic software and of course also by animal species (Birettoni et al. 2004, Carré et al. 1994, Goodwin 1998, Kuwahara et al. 2004, Steven and Pogwizd 1995, Suzuki et al. 1998).

Our previous experience confirms the common view that the Holter system can be used in mini-pig, sheep, dog, cat, rabbit, ferret, and rat. The mouse is too small (and therefore the interfering signals decrease the quality of the record). On the other hand, the pig is too big and restless in a non-anesthetized state for being studied by HECG. Number of papers describing HECG in sheep is very low - there is only one work by Reefmann et al. (2009). On the other hand, there are several articles on HECG use in the cow (Hagen et al. 
2005, Gyrax et al. 2008, Mohr et al. 2002, Schmied et al. 2008). It is a little bit surprising since sheep might represent an ideal animal for the HECG - it is big enough, it is quiet and has non-destructive behavior. In the countries with sheep-breeding tradition (such as Australia, New Zeeland and China) the sheep is often used in experimental projects.

In this article, our six-year experimental and clinical veterinary experience with classical Holter recorders routinely used in human clinical practice is overviewed.

\section{Equipment and methods}

Commonly single-channel or multi-channel recorders are routinely used in veterinary practice. Unfortunately, it is not possible to predict the quality of the final shape of ECG trace unless it is accomplished by one-channel Holter recorder. In our laboratory we have used IQmark Pacer (with software IQmark Workstation, Midmark, Great Britain) and Cardiolight Smart CRLASR-PD3E (SW Padsy, Medset Medizintechnik, Ggermany), both in three-channel version.

At present, the advantage of classical Holter electrocardiography is the digital system of recording on solid-state memory media (e.g. SD or CF card). We prefer recorders with built-in display enabling quality control without connection to personal computer (PC). It makes the installation of the system easier and the work more rational. Furthermore, there is no need to use a PC or to switch among PC applications demanding the hardware. On the other hand, the recorders without builtin display are much smaller lighter (by about one half) and they are less energy consuming. The ideal recorder enables multi-channel recording (usually three-channels), but with the possibility of easy switching to simple single-channel recording. These hardware requirements are essential if universal usage (more animal species and/or more types of experiments) is planned.

The system of leads is another extremely important issue. HECG recorders operate with bipolar leads in orthogonal system. The leads in the transversal (horizontal) or sagittal plane are the most suitable ones.

Routinely supplied software proved to be reliable in dogs and pigs, but the permanent control of the templates is always essential (Fig. 1).

Using the common software, the setting of the limits of tachycardia, asystole, and J-ST point distance in small animals is usually problematic. In cats, the relatively high but narrow $\mathrm{T}$ waves often fused with low $\mathrm{R}$ waves, resulting in an error of total numbers of QRS complexes, and consequently in the errors enlisted in templates. In cats and smaller mammals (rabbits, rats, and ferrets) with quite short QRS complexes and QT intervals, it is better to ignore ST/T elevations or depression and QT interval variability. It mainly concerns the cases in which detection algorithm of assessed parameters from manufacturer is not available or eventually where the software does not allow the modification of measuring of real values in monitored animal species.

Anesthesia is a mandatory part of HECG in rats. There is no place for sedation; general anesthesia is always necessary, but the mode is liberal and depends mainly on experimental protocol details or demands. From the size of ferret it is possible to record HECG in fully conscious animals. In uncooperative individuals, for system placement or removal, potent sedation $\left(\mathrm{alfa}_{2}-\right.$ agonists, benzodiazepines and various combinations) or short-term mono-anesthesia (inhalation anesthetics, propofol, etomidate) can be used.

\section{Experimental animals}

$\underline{\text { Rat }}$ - its size and namely its agility limits the usage of HECG in an conscious animal due to a frequent destruction of the device. Under general anesthesia, needle electrodes are easily applicable and markedly increase the quality of the record. Adhesive electrodes are unnecessarily expensive and the cost/benefit ratio is poor. Of course, crocodile clips and conductive gel can be used. However, with the increasing duration of recording, the quality of records declines due to corrosion and successive polarization of electrodes. The apex-base lead proved to be the ideal one for recording in the rat. It can be optimally obtained by connecting the different electrode $(+)$ to the left precordium or processus xiphoideus (pending on posture) and indifferent (-) electrode to the cervical slightly right paravertebral position. If multi-channel recorder is available, the rest of the bipolar electrodes should be placed approximately in the transversal plane, at the level of the $5^{\text {th }}$ or the $6^{\text {th }}$ rib. The regularly measured deflections, intervals and segments are well readable and clearly distinguishable and evaluable in the apex-base lead (Fig. 2, Fig. 3). 


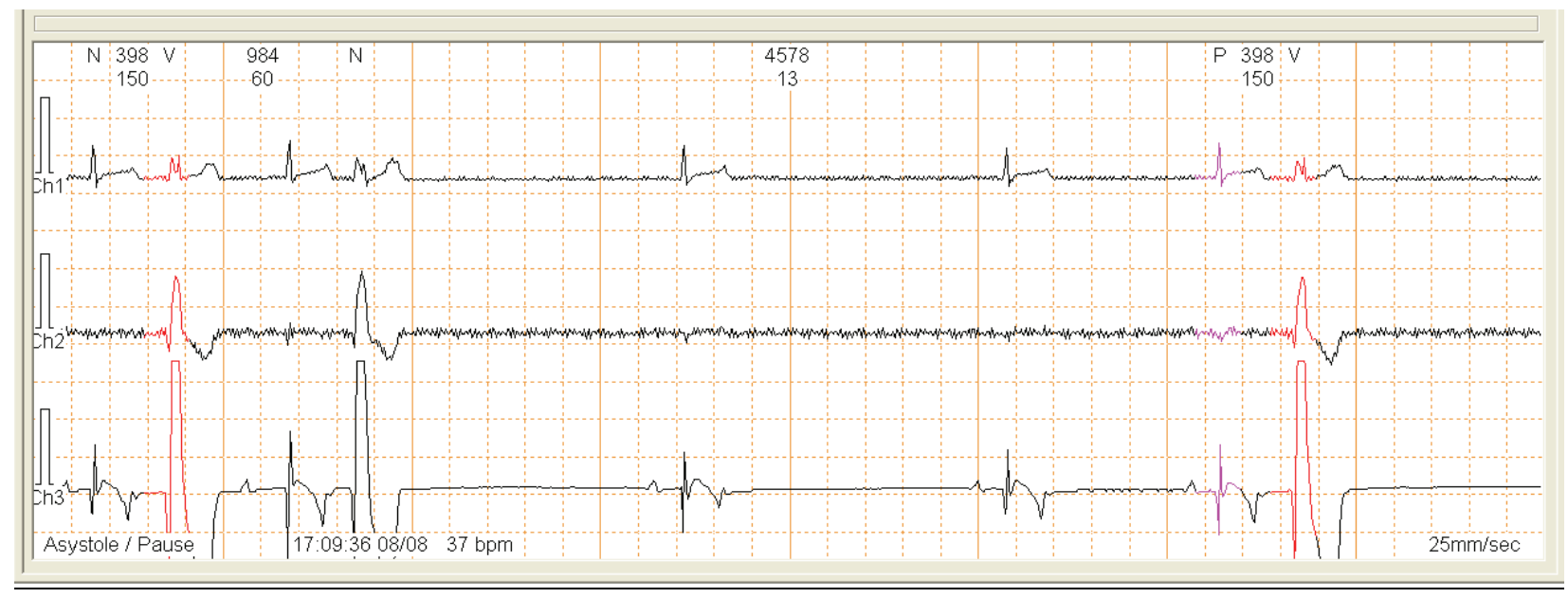

Fig. 1. Original record of window of HECG trace in dog. There is a problem to detect regular P-QRS-T formations by software, VPCs or normal complexes are detected randomly. This software error is not only episodic (in this record), but systematic; the result produced by the system is of no use and manual correction is needed. However, it means remarking of about 10,000-15,000 ignored complexes.
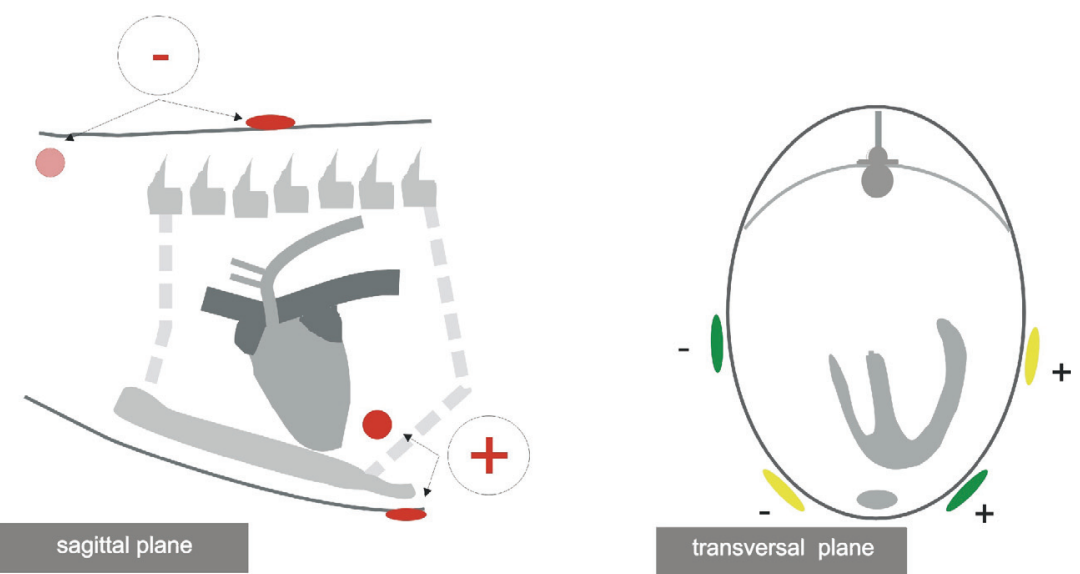

Fig. 2. Lead settings scheme in the 3 channel recorders: bipolar apex-basis lead on the sagittal plane and two bipolar leads in the transversal plane.

+ different/active electrode,

- indifferent/passive electrode.

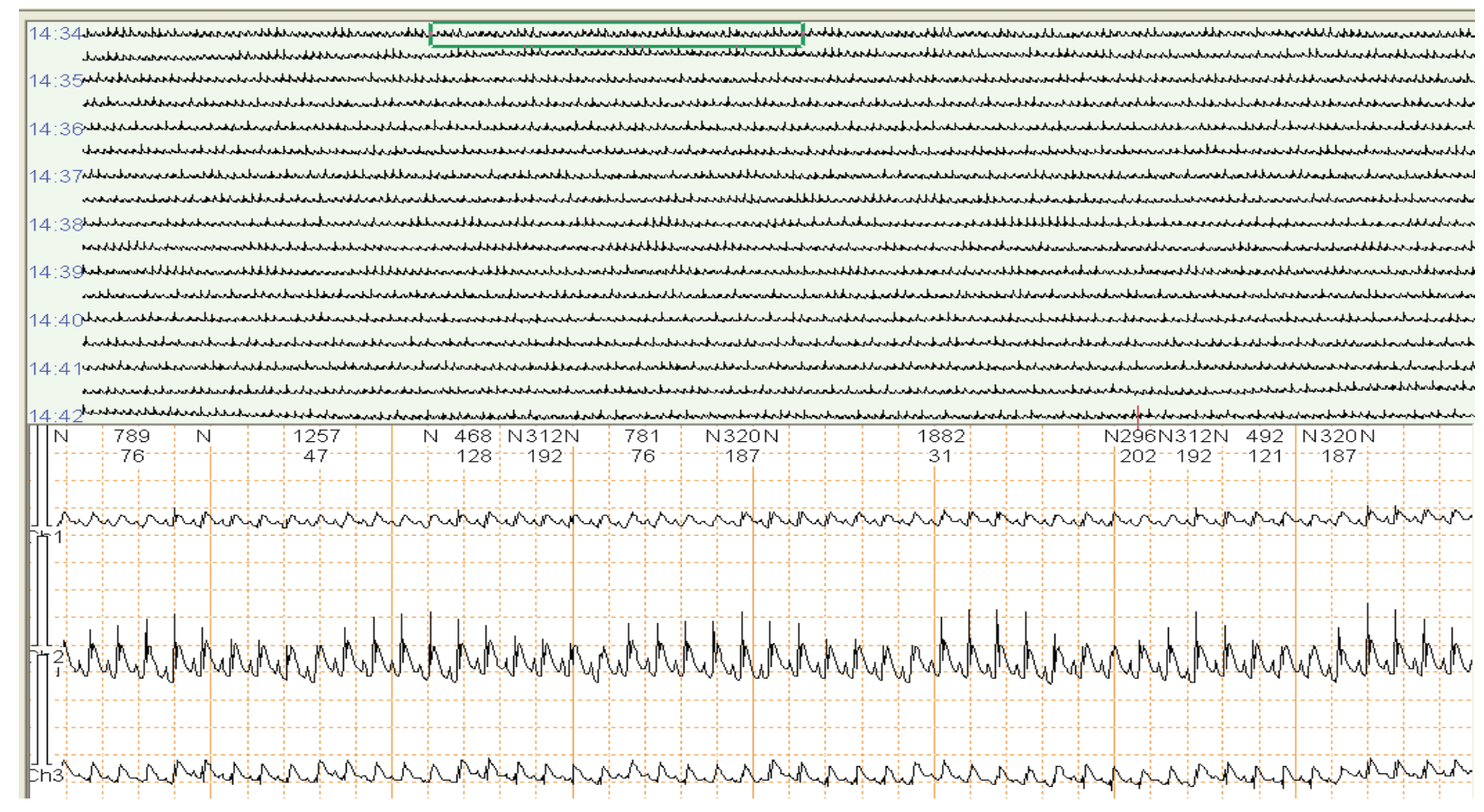

Fig. 3. Original record of HECG trace in the rat (animal under xylazine-diazepam sedation). Although curve quality is sufficient, automatic detection QRS complex fails. Nevertheless, manual heart rate measurement is easy. Lead connection according to Fig. 2. 


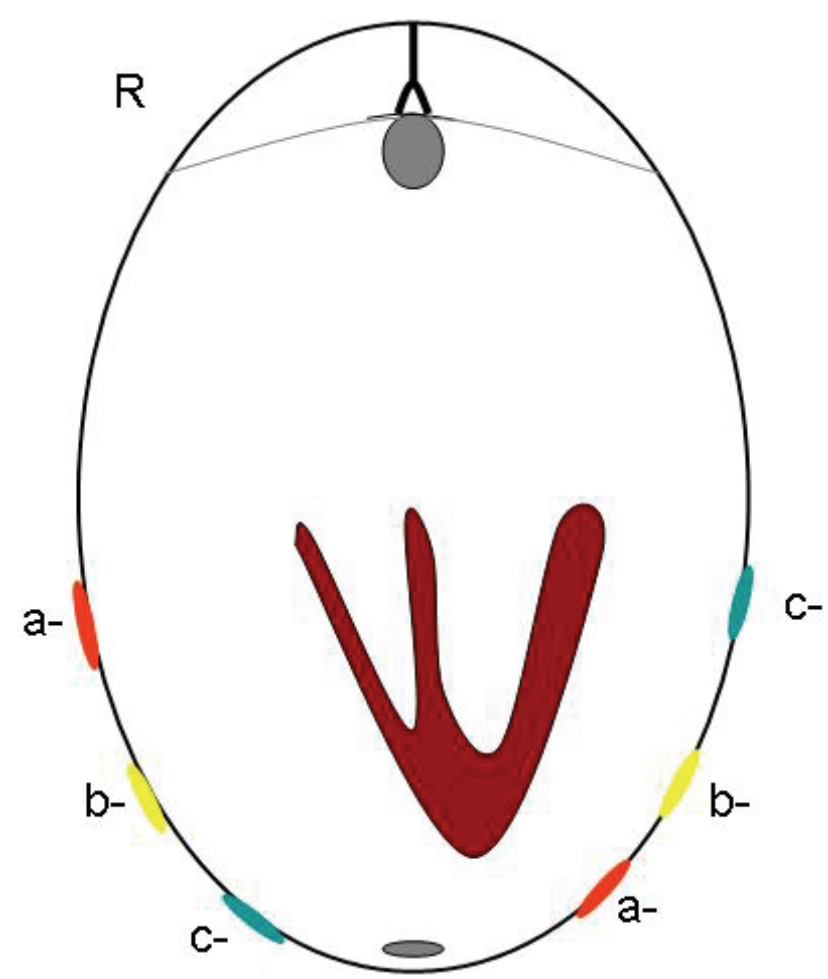

Fig. 4. Scheme of chest leaking places in bipolar leads in transversal plane.

Rabbit - may be adequately examined by HECG without permanent sedation, whether it is required by the experimental protocol or not. The system of leads and type of electrodes are selected in accordance with actual situation. If the experimental protocol requires total anesthesia, strategy of recording is identical to that described in the rat: the same needle electrodes and connections are used. That represents at least one apexbase lead and the rest is localized circularly above the $5^{\text {th }}$ and the $6^{\text {th }}$ ribs. In order to obtain the records in a conscious animal, all leads are placed in the transversal plane (Fig. 4, Fig. 5); adhesive electrodes designed for HECG may be used (they enable the fixation of conducting medium and protect against unwished pull).

Skin has to be shaved and degreased where the electrode should be glued-on. The cable is fixed by a plaster in one layer only and by a bandage for comfortable breathing. The recorder is fixed to the body by a bandage, too. The bandage is finished with a plaster (waterproof is the best). In the rabbit, the Elisabeth's collar should be employed in order to prevent the derangement of the recorder.

Ferret - cylindrical body shape and short limbs make fixation of the recorder more difficult in comparison with the rabbit. An auxiliary plaster overlap in marginal parts with direct fixation to the hair or shaved skin is recommended. Setting-up and off the recorder is recommended to be done in brief general anesthesia, otherwise it is a pain-staking process.

Cat, dog, mini-pig - mounting and dismounting the apparatus is relatively simple in socialized animals. Various nylon or linen jackets have been described that might serve as protection of the recorder and cables (Steven and Pogwizd 1995, Suzuki et al. 1998). Nevertheless, these products pay-off only in the case of using the same breed of the same age. Therefore, the bandage, although it is rather time-consuming (approximately $10 \mathrm{~min}$ ), is recommended. Because of the possibility of movement, the lead system should respect the anatomical differences in posture, shape of chest and location of the heart. Although we also tested the lead system described by Goodwin (1998) adapted from connections used in humans, we prefer the simple circular placing of electrodes over the left and right precordium in a transversal plane (Fig. 3). This lead system is stable in motion and enables the common evaluation of record.

Sheep - as the manipulation with experimental animal is concerned, sheep is the most comfortable species. The only necessary additional equipment is the wool cutting machine. The range of sheep breeds is large from miniature one to big meat breed. Mounting and dismounting of the recorder is simple - due to the woolfell the recording system excellently adheres and if the animal is limited spatially to prevent jumping, quality of record is wonderful.

\section{Discussion}

In our experimental practice, the HECG represents an auxiliary paraclinical investigation allowing us to work efficiently, especially in pilot studies - we can minimize the number of experimental animals. To be specific, our team deals with models of sepsis and septic shock in rats and rabbits. In such projects, it is fundamental to titrate the dose of infection in order to ensure that the septic shock develops on the second or the third day in the morning. Then the time-consuming sophisticated instrumental monitoring and treatment can proceed invariably during standard working hours. HECG help us in determination of breakpoint, where the decrease of blood pressure is not followed by increase of the heart rate any more. Blood pressure is monitored by oscillometric method or estimated from Doppler flow in pulmonary artery or aorta (Dembovská et al. 2008, 


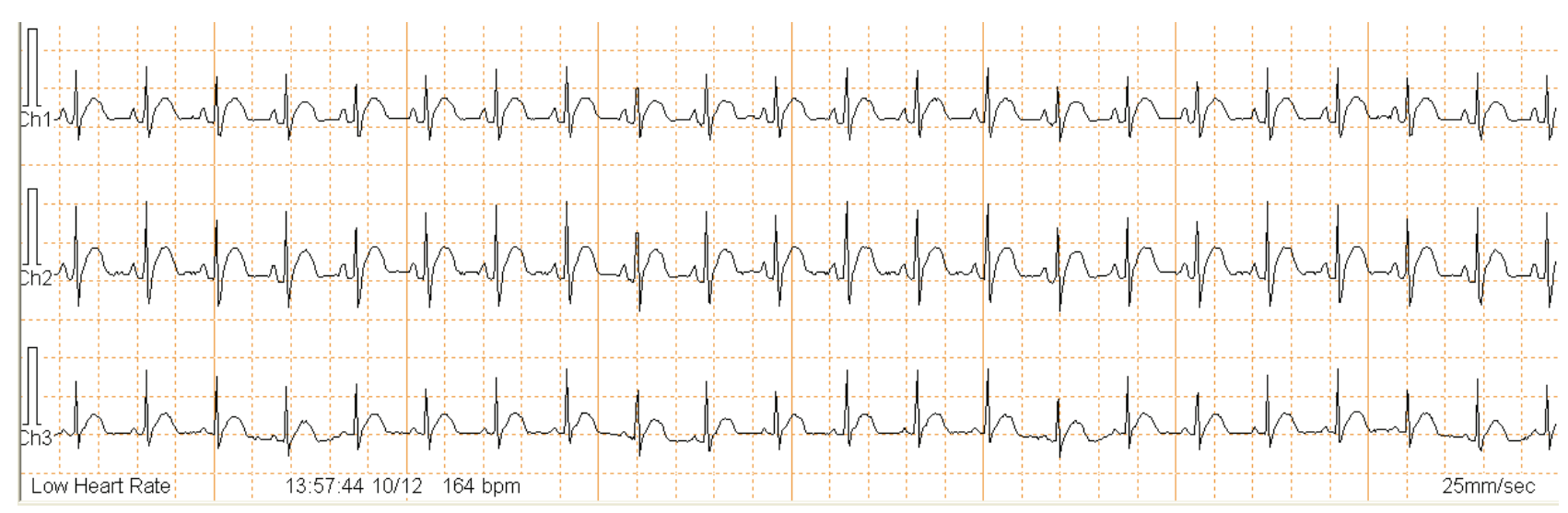

Fig. 5. Original record of HECG trace in the rabbit (awake animal). The quality of record is high and thus the automatic detection of QRS complex is exact. Lead connection according to Fig. 4.

Scheer et al. 2008). The HECG is also used in parallel with vital function monitoring, where laboratory technician marks into the protocol individual maneuvers (sampling, drug administration, change of device setting, etc.) or abnormal events (wake-up, failure of devices, obturation or cranking of catheter, and arrhythmias). Consequently, later it is easy to find and evaluate any of above mentioned events.

Another interesting topic is the determination of mean resting heart rate, which is possible by using the HECG. This parameter is important particularly for setting-up the resting rate in patients with permanent cardiac pacemaker (PCPM). Our results obtained in healthy dogs suggest that heart rates around 30 beats per minute during sleeping are common and asymptomatic also in small dogs with body weight of about $10 \mathrm{~kg}$. Veterinary electrocardiologic bible (Fox 1992) stipulates: "pace rate - small dogs and cats 100 beats/min, large dogs 75 to 80 beats/min", and this "law" is still repeated by Fox (1992) and Kienle (1998). According to this stipulation, in dogs, where the dual-chamber PCPM is implanted, the same resting heart rate ( 55 beats $/ \mathrm{min}$ ) was set up as it is used in humans ( $50-60$ beats/min), even if the body weight of these dogs varied between $20-30 \mathrm{~kg}$. Our experience is identical with the set-up data in 33 paced dogs (Hildebrant et al. 2009). In their group, the preset of lower pacing rate was $40-60$ beats $/ \mathrm{min}$. This observation is worthy of further examination, although it is against the commercial interest of companies producing PCPM's. Lower pacing rate namely extends effective life of the PCPM and thereby improves costefficiency for the payer/owner of the veterinary patient.

\section{Conclusions}

Holter electrocardiography is a simple and applicable method of monitoring the electrical activity of the heart in small animals. If the protocol of experiment does not preclude general anesthesia, it is easily applicable even in rats. In bigger animals, it is sometimes necessary to employ general anesthesia. The usage of bipolar leads in sagittal and transversal planes proved to be the most suitable, because of large amplitude of QRS complex and uncomplicated automatic analysis of the record.

\section{Conflict of Interest}

There is no conflict of interest.

\section{Acknowledgements}

The paper was supported by the grant IGA MZCR NR 9284-3/2007. We gratefully acknowledge Prof. M. Nováková and Prof. P. Bravený for generously providing helpful remarks and critical evaluations of the text.

\section{References}

BIRETTONI F, PORCIELLO F, RUECA F., FRUGANTI G: 24-hour ambulatory electrocardiography in the dog. Vet Res Commun 28: 323-325, 2004.

CARRÉ F, MAISON-BLANCHE P, MANSIER P, CHEVALIER B, CHARLOTTE N, DAKHLI T, COUMEL P, SWYNGHEDAUW B: Phenotypic determinants of heart rate variability in cardiac hypertrophy and failure. Eur Heart J 15 (Suppl D): 58-62, 1994. 
DEMBOVSKÁ K, SVOBODA P, SCHEER P, KANTOROVÁ I, TOMENENDALOVÁ J, ŘEHÁKOVÁ K, DOUBEK J: Model of septic shock induced by live E. coli (O18) in laboratory rat. Vet Med Czech 53: 153$163,2008$.

FOX PR: Special methods for treating arrhythmias: cardiopulmonary arrest and resuscitation, pacemaker therapy In: Manual of Canine and Feline Cardiology, TILLEY LP (ed), Saunders, St. Louis, 2008, pp 365-381.

GOODWIN JK: Holter monitoring and cardiac event recording. Vet Clin North Am Small Anim Pract 28: 1391-1408, 1998.

GYRAX L, NEUFFER I, KAUFMANN C, HAUSER R, WECHSLER B: Restlessness behaviour, heart rate and heartrate variability of dairy cows milked in two types of automatic milking systems and auto-tandem milking parlours. Appl Anim Behav Sci 28: 15-39, 2008.

HAGEN K, LANGBEIN J, SCHMEID C, LEXER D, WAIBLINGER S: Heart rate variability in dairy cows influences of breed and milking system. Physiol Behav 85: 195-204, 2005.

HILDEBRANT N, STERTMANN WA, WEHNER M, SCHNEIDER I, NEU H, SCHNEIDER M: Dual chamber pacemarker implantation in dogs with atrioventricular block. J Vet Intern Med 23: 31-38, 2009.

KIENLE RD: Interventional antiarrhytmic therapy. In: Small Animal Cardiovascular Medicine, KITTLESON MD, KIENLE RD (eds), Mosby, St. Louis, 1998, pp 525-538.

KUWAHARA M, TSUJINO Y, TSUBONE H, KUMAGAI E, TSUTSUMI H, TANIGAWA M: Effects of pair on diurnal rhythms of heart rate and heart rate variability in miniature swine. Exp Anim 53: 303-309, 2004.

MILLER MS, CALVERT CA: Special methods for analyzing arrhythmias. In: Essentials of Canine and Feline Electrocardiography, TILLEY LP (ed), Lea Febinger, Philadelphia, 1992, pp 289-319.

MOHR E, LANGBEIM J, NÜRNBERG G: Heart rate variability: a non-invasive approach to measure stress in calves and cows. Physiol Behav 75: 251-259, 2002.

REEFMANN N, BÜTIKOFER-KASZÄS F, WECHSLER BEAT, GYGAX L: Physiological expression of emotional reactions in sheep. Physiol Behav 98: 235-241, 2009.

SCHEER P, SVOBODA P, DOUBEK J, RADVANOVÁ J, RADVAN M, KANTOROVÁ I: How to follow hemodynamics in experimental septic shock of small animals. Hepatogastroenterology 55 (Suppl I): A319, 2008.

SCHMIED C, WAIBLINGER S, SCHARL T, LEISCH F, BOIVIN X: Stroking of different body regions by a human: effects on behaviour and heart rate of dairy cows. Appl Anim Behav Sci 109: 25-38, 2008.

SLEEPER MM: Special diagnostic techniques for evaluation of cardiac disease In: Manual of Canine and Feline Cardiology, TILLEY LP (ed), Saunders, St. Louis, 2008, pp 99-107.

STEVEN M, POGWIZD MD: Nonreentrant mechanisms underlyining spontaneous ventricular arrhythmias in model of nonischemic heart failure in rabbits. Circulation 92: 1034-1048, 1995.

SUZUKI A, TSUTSUMI H, KUSAKABE M, KUWAHARA M, SUGANO S, TANIGAWA M: Establishment of a 24hour electrocardiogram recording system using a Holter recorder for miniature swine. Lab Anim 32: 165-172, 1998. 